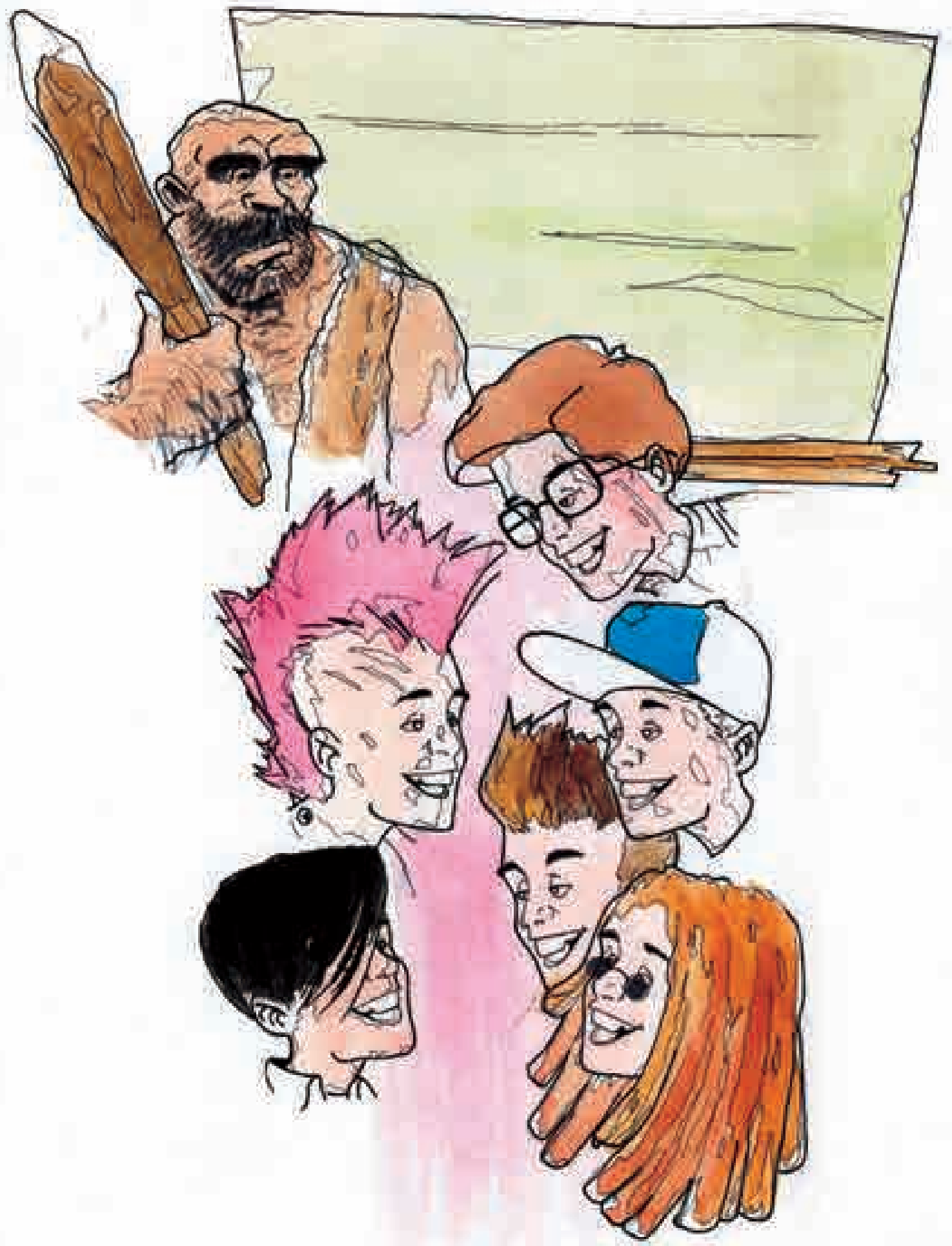

Ilustración 22. Los días que siguieron al Foro Educativo sus actividades se trenzaron en sus compromisos laborales, deberes educativos y oficios religiosos, así que hubo poco tiempo para el encuentro. No obstante, cada uno realizó sus consultas en torno a las pedagogías existentes, pero ninguna les satisfizo. La más próxima fue la pedagogía crítica, pero no los llenaba del todo. 


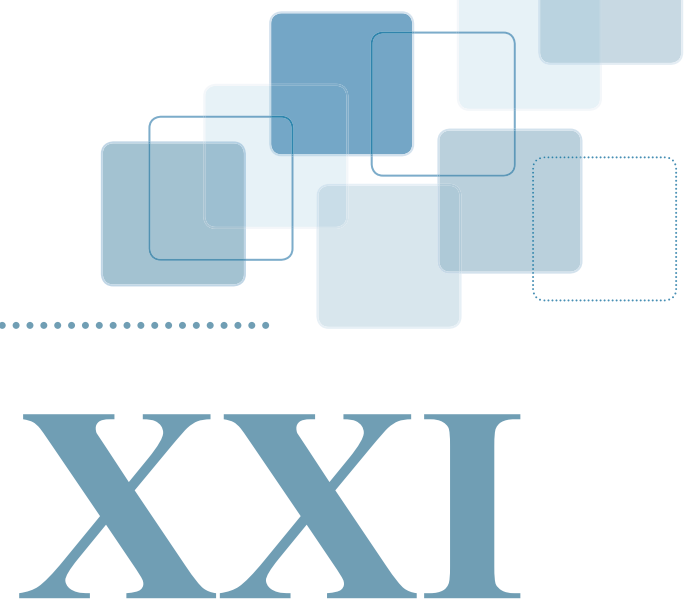

\section{HACIA UNA PEDAGOGÍA DE LO COTIDIANO}

Semanas después los frailes encontraron el espacio para volver a hablar de su trabajo de investigación. Los días que siguieron al Foro Educativo sus actividades se trenzaron en sus compromisos laborales, deberes educativos y oficios religiosos, así que hubo poco tiempo para el encuentro. No obstante, cada uno realizó sus consultas en torno a las pedagogías existentes, pero ninguna les satisfizo. La más próxima fue la pedagogía crítica, pero no los llenaba del todo.

-Tiene que haber una pedagogía que privilegie "el aprender" por encima del "enseñar" - dijo Luis Eduardo, que la ciudad, el barrio, la calle, el centro comercial y el ciberespacio sean campos de conocimientos y no áreas del saber, que traigan lo que acontece en la vida diaria de los jóvenes a la escuela y que esta convierta, esta fuerza subterránea, "lo cotidiano", en un poderoso caudal para producir saberes racionales.

— ¡No existe! —dijo Rubén Darío, desconsolado.

-Entonces, nos tocó inventarnos una pedagogía que sea capaz de hacerlo —acuñó Carlos Ariel. Un silencio profundo se apoderó del lugar. 
— ¿Pedagogía de lo cotidiano? —repuso Luis Eduardo.

— ¡Me gusta! —dijo Rubén Darío. Los relatos narrativos de los sujetos pedagógicos, las entrevistas realizadas y las ponencias de Fabián Sanabria, Andrés Argüello y Antanas Mockus en el Foro Educativo, nos conducen a crear una manera diferente de abordar las relaciones entre los sujetos pedagógicos.

\section{— ¡De acuerdo! —agregó Carlos Ariel.}

Los meses siguientes el trabajo de los frailes se centró en la construcción epistemológica y los principios de la nueva pedagogía. Por momentos se encontraban, compartían onces, tomaban el café, preguntaban a sus maestros en la maestría acerca de algunas dudas en torno a las pedagogías existentes y construían algunas directrices en torno a la pedagogía de lo cotidiano. Tres meses después del foro tuvieron la siguiente conversación:

- Si lo cotidiano siempre es nuevo, entonces, la pedagogía de lo cotidiano debe hacer de lo nuevo su valor principal —dijo Rubén Darío.

—“"El hombre es la única criatura que ha de ser educada" (Kant, 2004). -Interpeló Luis Eduardo. Existen organismos en la naturaleza con estructuras menos complejas que la de los seres humanos. Las tortugas, peces, ratones, gatos, perros, entre otros, nacen con la información genética necesaria para adaptarse al medio natural en tiempo record. El hombre, en cambio, "requiere un proceso de aprendizaje y la adquisición de información que no tiene al momento de nacer. Sin la ayuda de sus pares, el ser humano nunca podría completarse como individuo" (Arocena, 2012, p. 26). En la relación con los otros individuos el hombre construye -además de su individualidad-, el aprendizaje necesario para sobrevivir, así responde a la ausencia de la información genética que otros animales de estructuras menos complejas que las suyas si poseen. El encuentro con el otro es la metáfora de la sociedad. Kant ya intuía para 1777, que el hombre es el animal más indefenso a la hora de nacer, por eso creyó que debía ser educado. A través de la educación el hombre supera las dificultades genéticas que no posee al nacer. 
-Aprendiendo es como el hombre construye, edifica, diseña, agregó Carlos Ariel- todo el conocimiento que el hombre necesita para vivir y convivir con los otros. En consecuencia, la pedagogía de lo cotidiano debe hacer del aprendizaje la coordenada básica de la escuela del siglo XXI. Aprender es connatural al hombre y quizá sea esta capacidad la que nos distingue de los otros seres vivos. Aristóteles creía en el libro primero de su Metafisica que "Todos los hombres desean por naturaleza saber", suponiendo que el secreto de la educación no está en lo que se enseña sino en lo que se aprende. Ello recrea la famosa aporía de la docta ignorantia socrática "solo sé que nada sé", a través de la cual "nos hacemos conscientes de nuestra finitud y limitación" (Gadamer, 2000, p. 23), como afirma Gadamer. Aprendemos de los otros más que de nosotros mismos. Aprendimos de las generaciones precedentes el conjunto de estrategias que nos condujo a la conquista del espacio y a maravillarnos con el ciberespacio, pero también aprendimos a fabricar las armas más inimaginadas que ninguna especie viva ha sido capaz de inventar. Ambas las aprendimos de nuestra relación comunicativa con los demás. A. Montagu en Homb e y agresión afirmó, cuatro décadas atrás, que "ningún ser humano ha nacido nunca con impulsos agresivos u hostiles y ninguno se hace agresivo u hostil sin aprenderlo". Aprender, entonces, es muy peligroso, pues de la misma manera que nos garantiza la supervivencia ella misma supone la destrucción de la humanidad.

-Entonces, el principio fundamental de la pedagogía de lo cotidiano debe ser "Aprende más enseña menos" — dijo Rubén Darío. Este principio, es lo que ha hecho que Singapur, Finlandia y Corea del Sur, entre otros, hayan superado la era de la post-estandarización educativa (Hargreaves y Shirley, 2012, p. 12). El vector educativo de algunos países de la Organización para la Cooperación y el Desarrollo Económico - OCDE “Aprende más enseña menos" se opone tangencialmente a nuestro desgastado principio "evaluar para mejorar", porque la evaluación no es el centro del proceso escolar como erróneamente se ha creído en nuestro país. La pedagogía de lo cotidiano, invierte el ciclo gnoseológico clásico "enseñanza-aprendizaje” y rompe la ecuación 
"maestro-estudiante", ahora, con esta pedagogía, el nuevo ciclo se presenta bajo la fórmula "aprender y enseñar" y la relación entre los sujetos pedagógicos es con relación al aprendizaje.

-Ahora bien, un segundo principio de la pedagogía de lo cotidiano debe ser "aprender del otro" — repuso Carlos Ariel. Fabián Sanabria cree que "ser joven es tener la capacidad de escandalizar" y los jóvenes siempre han escandalizado, la hermenéutica de lo cotidiano da testimonio de ello: las nuevas generaciones tienen un código de gestos directos, expresiones y decires impúdicos y obscenos, construido a partir del desvanecimiento de todo lo que era absoluto y, expresado a través de una espontánea libertad sexual síndrome dionisíaco generacional. El mensaje que encierra este código "de lo grosero" se enmarca en toda una suerte de insultos comunicacionales y se instaura en las relaciones juveniles. El escarceo y el juego eróticos -invocación del hedonismo que crece con el siglo-, se desculpabiliza en la instantaneidad del gesto obsceno, en el ahora de la fiesta dionisiaca y en la fugacidad de lo grosero, siendo esta última, la expresión más desinhibida del esnobismo juvenil. Lipovetsky ha encontrado en estas incitaciones al hedonismo, una clara manifestación "del gusto por lo fácil y lo ligero, el gesto por lo erosivo y lo lúdico como consubstancialidades del deseo humano" (Lipovetsky, 2007, p. 24). Aprender del otro, es un acto de fe en él, H. Gadamer en La educación es educarse creía que "se aprende de aquellos que aprenden de uno", y la primera década del siglo XXI le confiere la razón, pues los jóvenes de hoy experimentan toda una serie de acontecimientos que ninguna generación conoció. Los jóvenes de hoy -pensando con Andrés Argüello- poseen una inteligencia cultural que hace que las personas logren acuerdos y entendimientos a nivel cognitivo, ético, estético y afectivo. Esta relación dialógica hace que cada individuo, cuando interpela al otro, lo interpelado se convierte en un texto para la construcción del conocimiento colectivo. "De aquí que las habilidades, modos de conocer, valoraciones y demás expresiones del pensamiento tiene cabida en el colectivo de aprendizaje" (Argüello y Mondragón, 2012, p. 89) aprender del otro implica entender que 
la nueva manera de concebir la relación con el conocimiento solo es posible a través de una relación horizontal fraterna.

—Larelación dialógica para la construcción discursiva del aprendizaje colectivo revaloriza lo que la pedagogía critica, específicamente, en Pedagogía de la autonomía de Paulo Freire, cuando este afirma que no hay docencia sin discencia —dijo Rubén Darío. Quiere decir esto, que aprender del otro pasa por un reconocimiento de él, para mi propia existencia. La pedagogía de lo cotidiano propone, entonces, aprender del otro, incluso de aquellos que aprenden de uno, porque la educación es un ejercicio circular, todos aprendemos de todos. Dominique Groux del Instituto Católico de París el pasado 19 de septiembre en la Universidad Santo Tomás afirmó que cuando somos conscientes de la existencia del otro, surge el momento en el que somos conscientes que vivimos separados de los otros, que existe un "otro" distinto a mí. La otredad es la expresión del abandono de la individualidad para reconocer lo distinto, lo diferente, es decir, al otro. La otredad reactualiza la vieja metáfora "cálzate los zapatos del otro", lo cual implica ponerse en el lugar del otro para ver qué tanto tallan en él sus zapatos.

Groux comparte con Bourdieu, la necesidad de reconocer al otro como algo distinto a mí. Es una empatía que se logra solo ante el otro, es la capacidad que solo se logra cuando se reconoce esta diferencia, así se valora y respeta al otro. En la otredad se encuentra lo que me hace falta, es el lugar donde se halla la otra parte que me hace falta a mí. Reconocer al otro es un acto humano: cuando reconozco al otro, este no tiene más camino que reconocerme a mí, el reconocimiento del otro es mi propio reconocimiento. Además, una vez que lo reconozca y que él me reconozca a mí, él no tiene oportunidad de agredirme. Entonces, la alteridad además de ser biunívoca también garantiza supervivencia mutua.

Todos estamos educándonos en la educación -asevera Groux-, lo cual hace pensar en la afirmación que H. Gadamer hiciera en 1998 "educar es educarse". En el otro me educo a la vez que el otro se educa en mí. La educación cobra otros ribetes, no educo sino que me educo. Yo soy 
yo, en la medida que me reconozco en el otro, este es el espejo que me hace verme a mí mismo. El otro es la fuente mientras yo soy Narciso. La interculturalidad es el paso hacia la alteridad, es la capacidad-dice Groux - de aceptar lo novedoso y exótico que el otro me presenta. La novedad y lo exótico debe ser reconocido en lo religioso, lo cultural, lo político y lo social. En la otredad y en la diferencia, consecuencia de un mundo cada vez más plural, el lenguaje cobra mayor importancia. Hablar y escuchar son importantes habilidades que tiene que desarrollar la pedagogía de lo cotidiano para entender y reconocer al otro. Entre tanto, escribir y leer son otras habilidades que fijan en el papel las múltiples voces - narrativas, según P. Ricoeur- que dan cuenta de la existencia de otras personas, que necesitan que yo las reconozca para que ellas me puedan reconocer a mí.

- La pedagogía de lo cotidiano rompe así con la estructura vertical descendente de la escuela moderna -agregó Luis Eduardo. Esta nueva pedagogía propone una estructura escolar flexible, en la que las formas de coexistir en el espacio escolar, así como los currículos y los planes de estudio se construyen conjuntamente con el otro, es decir, con todos los sujetos pedagógicos. Paulo Freire, en Pedagogía de la esperanza, había dimensionado la importancia de construir los currículos con la participación del otro, de aquel al cual se desea enseñar ${ }^{17}$. En 1968 H. McLuhan, en El aula sin muros, afirmó "que la mayor parte de la enseñanza tiene lugar fuera de la escuela". Con ello, quiero decir que la pedagogía de lo cotidiano deja en entredicho las fronteras de la escuela y hace de "la topología de lo cotidiano", así como de "la cronología de lo cotidiano" un importante referente para la educación del nuevo milenio.

-Desde la pedagogía de lo cotidiano se piensa la escuela, el sistema educativo, la educación, la ciudad, el barrio, la calle, el centro comercial,

17 Sin embargo, Freire no ha sido el único, revísese por ejemplo La sociedad desescolarizada (1971) de Iván Illich y $S$ qi ere ser rico y feliz no vaya a la escuela de Robert Kiyosaki, son referentes importantes en la pérdida de la hegemonía de la escuela. 
el ciberespacio y todas las relaciones y acontecimientos que en ellos se suscitan, de una manera diferente - dijo Carlos Ariel. Es decir, como un escenario de formación, que además de ser novedoso, es un amplio campo de conocimiento en el que se pueden articular todas las islas disciplinarias -áreas del saber-, y un poderoso caudal con el cual los sujetos pedagógicos pueden elaborar otro tipo de relación con el saber.

- "La escuela ha perdido la hegemonía sobre la información" (McLuhan, 1968, p. 235) -interpeló Rubén Darío-, lo que ha hecho que los maestros se hayan convertido en marineros que enseñan con viejos mapas, a grumetes que surcan autopistas de bits orientados por GPS. Los jóvenes de hoy ya no aprenden ni con maquetas, ni con frisos, ni con tareas sin sentido. A. Piscitelli (2009); se ha referido a la forma como aprenden "los nativos digitales": estos lo hacen aprendiendo "cacharreando", nosotros, en cambio, "migrantes digitales", aprendemos leyendo el manual de instrucciones de todos los dispositivos y objetos tecnológicos que adquirimos. Los jóvenes de hoy aprenden en la ciudad, el barrio, la calle, el centro comercial, el bar, la taberna, entre otros, el conocimiento necesario para vivir el día a día, la escuela y su corolario; los maestros deben desmontarse de la idea de enseñar y aprender como lo hacen sus estudiantes. Estos aprenden a un ritmo vertiginoso y producen otro tipo de saber que aquellos desconocen. Piscitelli cree que el desafío de los "inmigrantes" es doble: "hay que aprender cosas nuevas, y tenemos que enseñar las cosas viejas de un modo nuevo... pero quizá lo más desafiante es enseñar lo viejo con ojos nuevos" (Piscitelli, 2006). Los maestros están enseñando, como afirma Piscitelli (2006), "teorías y conceptos que no son significativos, para una época que ya fue y con modalidades anacrónicas que cada vez interesa menos" (Piscitelli, 2009, p. 178). La escuela se centró en enseñar desde el pasado moderno, frío, aburrido y racional un futuro incierto, impensado, que pasará cuenta de cobro, desdeñando el presente campeante de una generación de jóvenes que, de la misma manera, desestima el pretérito y lo que ha de llegar, para vivir el presente, aquí y ahora. 
Hay tanta información que ya no sabemos qué hacer con ella hizo una breve pausa y continuó. Zalamea cree que "nos encontramos desorientados, no por ausencia de conocimiento sino por exceso del mismo" (2004, p. 41), pero esta abundancia de información nos hace seres inmóviles, porque como dice Zalamea:

Hay tanta información disponible, tantos modos de conseguirla, tantas perspectivas para interpretarla, que la misma saturación de la información nos lleva a la inmovilidad. Sin poder distanciarnos de la proliferación de los datos, nos vemos como un punto más entre millones de puntos incapaces de elevarnos y de moldear la información, de correlacionar, distinguir, separar, jerarquizar (2004, p. 41).

Entonces, la pedagogía de lo cotidiano debe crear las bases para interpretar la realidad y moldear la información para construir el saber que las nuevas generaciones necesitan. Por último, en 1970 el futurista Alvin Toffler en El shock del futuro, citando a Herbert Gerjuoy, recordaba que "los analfabetos del siglo XXI no serán aquellos que no sepan leer ni escribir, sino aquellos que no sepan aprender, desaprender y reaprender".

-Eso solo podemos hacerlo a través de una hermenéutica de lo cotidiano, es decir, el tercer gran principio de la pedagogía de lo cotidiano - repuso Luis Eduardo. O sea, la pedagogía de lo cotidiano debe trabajar profundamente en las maneras de interpretar y comprender la realidad para hacer más atractivo los currículos. El reencantamiento del mundo y la vida de todos los días, son indicios del optimismo juvenil y razones que suponen el ocaso de los postulados racionales en beneficio de un compartir fraterno. La horizontalidad de los reagrupamientos juveniles ve emerger el $\mathrm{hm}$ o aeq alis en el que la proximidad al otro se realiza entre iguales, rompiendo, con ello, la verticalidad racional de las relaciones adultas. Lipovetsky encuentra en esta nueva expresión de juntarse un principio de "autonomía individual", suerte de mutación antropológica del individualismo humano. De esta manera, para Lipovetsky "Narciso se pone en órbita y es la metáfora de la vida asumida sin imperativos categóricos" (1990, p. 72), renuncia y 
fuga que invierte las normas instituidas para vivir la desmoralización de la vida en lo que Lipovetsky llama narcisismo colectivo. La entrega al compartir fraterno se convierte -según M. Maffesoli- en la dimensión afectiva y sensible de las relaciones sociales.

El narcisismo colectivo es "un perfil inédito del individuo en sus relaciones con él mismo, con su cuerpo y con los demás" (Lipovetsky, 2007, p. 26). Los lazos que se establecen en la taberna, las calles, el barrio, las plazoletas de los grandes centros comerciales, los conciertos, los after party, los chats room, en Facebook, Twitter, Messenger y tantas otras comunidades virtuales, son epifanías de la afinidad de intereses que engendra la vida colectiva y desata la pérdida del sujeto para fundirse en un cuerpo colectivo, porque "lo propio de la vivencia es poner el acento en la dimensión comunitaria de la vida social" (Maffesoli, 1996, p. 37).

En 1921, Weber incluía como características el aspecto fugaz, flotante y cambiante de las comunidades emocionales en respuesta a los valores instituidos por el racionalismo. Pere-Oriol siguiendo el análisis que Maffesoli realiza en El tiempo de las tribs, afirma que las comunidades emocionales, sujetos a la moda y a las relaciones efímeras fundamentadas en la comunión de emociones, se yerguen en oposición a las comunidades racionales de espíritu civilizador y progresista. Las nuevas comunidades, la vida colectiva y "las nuevas formas de agregaciones juveniles comparten una actividad y una actitud de sensaciones fuertes y le confieren a la existencia humana un contagio emocional, suerte de mensaje afectivo que nos recuerda el sentido ancestral del animal político" (Pere-Oriol, 1997, p. 12), que vive en comunidad generando una euforia vivida en la vida cotidiana de las nuevas generaciones.

"El contagio del sentimiento o de la emoción vivida en común liga al espacio próximo, al otro, al par, al que es semejante, en una relación de igualdad constitutiva de los grupos juveniles" (Maffesoli, 2004, p. 31). Constante búsqueda de compañía, fuerza atrayente que arroja a los 
jóvenes a fundirse en un devenir eterno con los otros, y manifestación colectiva de sensibilidad creciente que los sitúa en distintos entornos urbanos, los encuentros en los chats room, Facebook o Messenger, entre otros. De esta manera, el paradigma estético, metáfora de las comunidades emocionales explica el aspecto efímero y la composición cambiante del territorio, la pertenencia a una tribu, la moda y las costumbres tribales y la inscripción local a estas comunidades, a la vez que, manifiesta el abandono de lo racional en beneficio de la despolitización de las ideologías tribales.

- La hermenéutica de la vida cotidiana también revela que la vida juvenil se atrincheró en el presente - dijo Carlos Ariel. Asistimos a lo que Zalamea llama la "meticulosa destrucción de los tiempos y de las perspectivas lineales" (Zalamea, 2004, p. 121). De esta manera, se entiende porqué hoy en el universo juvenil existe un afán por vivir el presente a través de una ética hedonista que los aleja de las utopías, de las luchas y de las protestas, entregándose al culto de las emociones. Las ideologías han sufrido el mismo destino del tribalismo clásico, el notario del deseo ha firmado su defunción. El tiempo y el espacio, y pensando como Octavio Paz, "dejan de ser sucesión y vuelven a ser lo que fueron, un presente y un pasado en donde al fin se reconcilian" (Paz, 1981, p. 102). En esta misma dirección, G. Lipovetsky ha manifestado que la vida en presente ha remplazado las expectativas del futuro y el hedonismo a las militancias políticas. Esta afirmación encuentra estrecha relación con lo que Rossana Reguillo denomina "la incertidumbre y la fuga del futuro" o lo que para M. Maffesoli es el presentísmo por el que transitan las nuevas generaciones. La renuncia al futuro desideologiza el presente, desrracionaliza las costumbres y llena de emociones la vida cotidiana, expresión de aventura y principio en el que la fuga de sí se funde en el otro en una relación vital que le da sentido al vivir juntos.

—La desideologización de las ideas abstractas permuta hacia la emocionalización de la vida en una dinámica que invierte la máxima cartesiana: "Existo, luego pienso" — dijo Rubén Darío. La existencia se expresa ahora a través de lo sensual y dionisiaco, en detrimento de 
lo apolínico racional haciendo del presente la fuente de la que beben las nuevas generaciones. La apropiación de cada uno de los espacios urbanos, escuchar y bailar ritmos musicales que desenvainan lo primitivo corporal y desatan los deseos, los atuendos sicodélicos, el cuerpo convertido en vitrina en la que se exhiben toda suerte de colores y perforaciones, la exhibición pública de ombligos y parte de las zonas pudendas y mostrar en un acto sugestivo la ropa interior multicolor, son características de la emocionalidad con que se pavonea la existencia juvenil.

La energía vital que caracteriza a las nuevas agrupaciones jóvenes revela el desgaste y la saturación de los discursos racionales. El vitalismo juvenil late agitando lo abstracto y desdeñando la verticalidad descendente en la que se establecían las relaciones humanas, para vivir la vida en proyección horizontal fraterna, símbolo y expresión del $\mathrm{hm} o$ aeqal is. Maffesoli refiriéndose a este vitalismo y al derroche de energía afirma que tal respuesta vital "es una alternativa de resistencia a la forma oficial de vivir la vida y los valores instituidos por la modernidad" (Maffesoli, 2004, p. 31).

Toda esta fuerza subterránea es una vertiginosa explosión festiva y divertida en la que se vive la vida de todos los días, saber dionisiaco que alegra las calles urbanas y se impone colosalmente en las plazoletas de los centros comerciales. Vivir el presente, sin ataduras, en una relación de iguales en la que la vida grupal, narcisismo colectivo, comunidad emocional o simplemente vivir el placer de estar juntos, se constituye en el abandono por el futuro. Esta vitalidad se traslada hacia el territorio virtual y es el ciberespacio, otra de las epifanías de una vida que se vive en común y en estrecha relación con el otro en un sentido horizontal fraterno, porque tal horizontalidad no juzga, ni cuestiona o descalifica sino que en ella se acepta al otro tal cual como es.

La hermenéutica de lo cotidiano debe conducirnos a identificar "la desorientación del mundo contemporáneo" - de la cual se refiere Fernando Zalamea-, para que los sujetos pedagógicos asuman 
críticamente su realidad y sean capaces de transformarla. En consecuencia, la pedagogía de lo cotidiano. Aunque siempre esté enfrentada a lo actual, a lo novedoso y al cambio eterno que se presentan en las relaciones humanas no puede "renunciar de entrada a construir nuevas herramientas para cartografiar la cambiante topografía del mundo contemporáneo" (Zalamea, 2004, p. 27). Esta es la naturaleza irrefutable de la pedagogía de lo cotidiano, enfrentarse siempre a lo nuevo, sin amarras ni ataduras o señales que indiquen el camino por seguir. Son tantos y tan variados los acontecimientos cotidianos, que esta pedagogía tiene que valerse de la hermenéutica de lo cotidiano para decodificar, interpretar, reinterpretar críticamente la realidad, solo así, los sujetos pedagógicos pueden aprender, como Penélope, a tejer la urdimbre social con espíritu crítico.

-Los tres principios anteriores: "aprende más enseña menos", "aprender del otro" y "hermenéutica de lo cotidiano" hacen pensar que la distribución de los saberes y contenidos en áreas, no es suficiente dijo Luis Eduardo. Las áreas se convirtieron en islas disciplinarias que se preocupan solo por un fragmento del horizonte, se tenía la esperanza que los estudiantes -luego de ver ocho o nueve de estas, consideradas fundamentales- pudieran tejer con la paciencia de Penélope y con los hilos de Ariadna, el tejido completo del horizonte. Sin embargo, no fue subirlo así.

—¿Qué propones? —interpeló Rubén Darío.

— ¡Campos de conocimiento! —agregó Luis Eduardo. El horizonte de lo cotidiano no es uniforme, en él se producen cruces, transgresiones, mixturas y reinterpretaciones de la realidad.

- La pedagogía de lo cotidiano no solo debe aprender a escuchar el pensamiento de la plaza pública — pensando en las Historias florentinas de Maquiavelo, - intervino Carlos Ariel, sino que también debe aprender a decodificar, lo que se dice en el parque, la calle, el centro comercial, la taberna, el bar, el chat, el muro de Facebook, en What sApp, entre otros. 
-Entonces, el cuarto principio de la pedagogía de lo cotidiano son los campos de conocimientos - dijo Luis Eduardo. La ciudad: el barrio, el ciberespacio, la calle y el centro comercial son los cuatro grandes campos del conocimiento que necesita la pedagogía de lo cotidiano para desarrollarse. Aunque ella misma constituye un campo de conocimiento por sí sola. La noción de campo -conjuntamente con la de habitus y capital-, forman parte del sistema categorial de la obra del sociólogo francés Pierre Bourdieu. Estos tres conceptos se inscriben dentro de la metáfora espacial, tan característica en la obra de este sociólogo. Según Bourdieu el campo se tiene que pensar en forma relacional porque lo único que existe en el mundo social son relaciones:

En términos analíticos, un campo puede definirse como una trama o configuración de relaciones objetivas entre posiciones. Esas posiciones se definen objetivamente en su existencia y en las determinaciones que imponen a sus ocupantes, agentes o instituciones, por su situación (situs) actual y potencial en la estructura de la distribución de las diferentes especies de poder (o de capital), cuya disposición comanda el acceso a los beneficios específicos que están en juego en el campo, y, al mismo tiempo, por sus relaciones objetivas con las otras posiciones (dominación, subordinación, homología, etc. ${ }^{18}$

Desde esta perspectiva, el campo es un espacio simbólico y cultural, suerte de metáfora espacial que hace del espacio social algo fluido, dinámico y en constante progresión. Esta condición dinámica hace que los actores del campo entren con frecuencia en conflicto por las riquezas que este ofrece, según Bourdieu, existen múltiples campos: el campo artístico, el campo religioso, el campo económico, que obedecen a lógicas diferentes. En sus estudios sobre los fenómenos socioculturales de las sociedades contemporáneas, Bourdieu afirma que la sociedad es vista como "un conjunto de campos relacionados entre sí, y a la vez,

18 Entrevista del Director de Estudios en la Ecole desd Hautes Etudes en Sciences Sociales a Pierre Bourdieu: La lógica de los campos: Hab tus y capital. En: http://ssociologos.com/2013/06/23/ entrevista-a-pierre-bourdieu-la-logica-de-los-campos-habitus-y-capital/. Recuperado el 03 de enero de 2014. 
relativamente autónomos". De esta manera, "los campos tienden a especializarse en temas, sectores y aspectos de la vida sociales que van conformando lo que Bourdieu llamó estructuras estructurantes, esto es, espacios propensos a la incorporación de nociones y la producción de sentidos" (2014).

Hablar de campo es pensar relacionalmente. El campo es como un juego, porque según Bourdieu:

Los jugadores entran en el juego se oponen, a veces ferozmente, sólo porque tienen en común el atribuir al juego y a las apuestas una creencia (doxa), un reconocimiento que escapa al cuestionamiento (los jugadores aceptan, por el hecho de jugar el juego, y no por un "contrato", que vale la pena jugar el juego) y esta connivencia está en el principio de su competición y de sus conflictos (Bourdieu, 2013).

Si el campo es el escolar, entonces los sujetos pedagógicos representan a cada uno de los jugadores. Cada uno de estos ha aceptado jugar el juego de la escuela, no obstante sus convicciones y creencias, estas son necesarias y el camino que los conducirá al conflicto. Cada jugador posee un número indeterminado de fichas, capital -tal vez, vale decir, el conjunto de creencias, saberes y conocimientos que los hace participar en el campo escolar-. Al iniciar el juego escolar, aparecen las relaciones de fuerza, cada uno de los jugadores esgrime sus fortalezas, estrategias y jugadas maestras, definiendo así, la estructura del campo. En palabras de Bourdieu, sería lo siguiente:

Se puede imaginar que cada jugador tiene delante de sí pilas de fichas de diferentes colores, correspondientes a las diferentes especies de capital que posee, de manera tal que su fuerza relativa en el juego, su posición en el espacio de juego, y también sus estrategias de juego, lo que se llama en francés su "juego" (jeu), los golpes, más o menos riesgosos, más o menos prudentes, más o menos subversivos o conservadores que emprende dependen al mismo tiempo del volumen global (Bourdieu, 2013). 
La configuración de la estructura del campo está determinada por la dinámica de este. Las diferentes fuerzas que operan en el campo y las diferencias específicas que subsisten entre ellas, son el capital específico del campo.

El habitus, en la obra de Bourdieu, es "concebido como un proceso de socialización desde la infancia, que simultáneamente es generado por estructuras objetivas a la vez que, son generadoras de esquemas de conductas y prácticas sociales" Chihu (s.f.). "La teoría de los campos en Pierre Bourdieu”. Según Bourdieu, el campo es estructuralista y el habitus constructivista. En este los esquemas de percepción y acción constituyen el habitus, en tanto aquel, entiende que en la sociedad existen estructuras objetivas independientes de la voluntad de los individuos -la economía, política, la ciudad, la escuela, la calle, entre otros-. Aunque tienen el poder de determinar las prácticas y representaciones individuales. De esta manera, el habitus hace pensar, en aquel estudiante que se resiste a cortarse el cabello, se tatúa el cuerpo y usa piercings, al que la sociedad escolar no dudará un solo instante en tipificarlo como "chico rebelde". Esta condición rebelde aunada a sus fuerzas -saberes, creencias, críticas y vivencias-, es decir, capital, lo colocan en condición de subordinado o subordinador en el campoescuela. El habitus también sirve para explicar por qué las personas se comportan de tal o cual forma, cuando comparten el mismo o diferente entorno social.

Ahora bien, cada vez toma más fuerza la idea de "crear, paralelamente a la escuela, otros medios y entornos educativos" (Trilla, 2003, p. 17), por lo que pensar en "la topología de lo cotidiano" como elementos constitutivos escolares, distintos de los tradicionales, no es nada descabellado. Pensar en los campos de conocimiento significa superar las fronteras disciplinares, promete adentrarse en diálogo de saberes interdisciplinar, multidisciplinar y pluridisciplinar y hace de la pedagogía de lo cotidiano un importante instrumento para el pegamento de las situaciones diarias con el saber científico y racional. Aunque no es tan novedoso incursionar en los campos de conocimientos, pues 
Bolivia inició desde el 2012 una profunda reforma educativa a través de la Ley Avelino Siñani-Elizardo Pérez, que pretende eliminar el concepto clásico de áreas del saber -aunque estas mismas constituyen un campo de saber pero específico-. Los campos de saber y conocimiento que operan actualmente en Bolivia son:

- Cosmos y pensamiento.

- Comunidad y sociedad.

- Vida, tierra y territorio.

- Ciencia, tecnología y producción.

En Colombia, el caso más conocido es el que adelanta la Universidad Distrital de Bogotá, en el que se "reconoce la diversidad epistémica y metodológica de los campos, comprendida como fortaleza para enriquecer los lenguajes, los saberes y los contextos del mundo social, científico, tecnológico, físico y natural". Los campos de conocimiento en esta importante alma máter son:

- Ciencias Ambientales de la Tierra y la Salud.

- Ciencias Sociales, Humanas y Arte.

- Ciencias y Tecnologías.

Como se puede ver, la propuesta boliviana pretende agrupar grandes espacios sociales, mientras que la UniversidadDistrital pretende aglutinar disciplinas. Nuestros hallazgos permiten suponer que la pedagogía de lo cotidiano se sentiría más conforme con la ofrecida por el Estado boliviano. Por último, la pedagogía de lo cotidiano con la apropiación de campos de conocimiento, además de privilegiar un diálogo de saberes interdisciplinarios, pluridisciplinarios y multidisciplinarios, se adentra al campo de la innovación y de la investigación.

- La ciudad: el barrio, el ciberespacio, la calle y el centro comercial pueden ser otros universos educativos diferentes - dijo Carlos Ariel. 
-Creo, entonces -interpeló Rubén Darío- que los campos de conocimientos con los cuales se desarrolla la pedagogía de lo cotidiano son:

- El barrio.

- La calle.

- El centro comercial.

- El ciberespacio.

Cada uno de estos campos constituye las partes de un todo: el campo ciudad educadora. Sin embargo -como en la teoría sistémica de N. Luhmann-, las partes no son más importantes que el todo, ni este más importante que aquellas.

Luis Eduardo y Carlos Ariel estuvieron de acuerdo. Luego pactaron trabajar en la delimitación conceptual y epistemológica de los campos y se despidieron. 
